\title{
Efficient Approach for Dielectric Permittivity Measurements of Liquids Adopting a 3D-Printed Cavity Resonator
}

\author{
Giulia Maria Rocco ${ }^{1}$, Paweł Barmuta ${ }^{2,3}$, Xiue Bao ${ }^{2}$, Dominique Schreurs ${ }^{2}$, and Maurizio Bozzi ${ }^{1}$ \\ ${ }^{1}$ Department of Electrical, Computer, and Biomedical Engineering, University of Pavia, Pavia, Italy \\ ${ }^{2}$ WAVECORE Division of Department of Electrical Engineering (ESAT), University of Leuven, Leuven, Belgium \\ ${ }^{3}$ Institute of Electronics, Warsaw University of Technology, Warszawa, Poland
}

\section{Running title:}

Efficient liquid permittivity measurements

\section{Correspondence}

Dominique Schreurs

ESAT-WAVECORE, University of Leuven, Leuven, Belgium

e-mail: dominique.schreurs@kuleuven.be

\begin{abstract}
This paper presents an operator-friendly and cost-efficient approach to characterize the dielectric permittivity of liquids flowing in narrow tubes, such as drainage tubes used as part of the wound healing process in hospitals. The approach is based on an oscillator such that the characterization can be achieved with minimal operator (e.g., nurse) training. The oscillator's frequency is set by a 3D-printed cavity resonator as to offer flexibility for the tube line to pass through. The oscillator design had to be tailored to ensure the oscillation condition to be met over a wide range of varying liquid compositions passing through the 3D-printed cavity resonator. The design and results of a proof-ofconcept implementation are presented and discussed.
\end{abstract}

\section{Keywords}

3D-printing, fluidic sensor, permittivity measurements, resonant cavity, oscillator 


\title{
Efficient Approach for Dielectric Permittivity Measurements of Liquids Adopting a 3D-Printed Cavity Resonator
}

\author{
Giulia Maria Rocco ${ }^{1}$, Paweł Barmuta ${ }^{2,3}$, Xiue Bao ${ }^{2}$, Dominique Schreurs ${ }^{2}$, and Maurizio Bozzi ${ }^{1}$ \\ ${ }^{1}$ Department of Electrical, Computer, and Biomedical Engineering, University of Pavia, Pavia, Italy \\ ${ }^{2}$ WAVECORE Division of Department of Electrical Engineering (ESAT), University of Leuven, Leuven, Belgium \\ ${ }^{3}$ Institute of Electronics, Warsaw University of Technology, Warszawa, Poland
}

\begin{abstract}
This paper presents an operator-friendly and cost-efficient approach to characterize the dielectric permittivity of liquids flowing in narrow tubes, such as drainage tubes used as part of the wound healing process in hospitals. The approach is based on an oscillator such that the characterization can be achieved with minimal operator (e.g., nurse) training. The oscillator's frequency is set by a 3D-printed cavity resonator as to offer flexibility for the tube line to pass through. The oscillator design had to be tailored to ensure the oscillation condition to be met over a wide range of varying liquid compositions passing through the 3D-printed cavity resonator. The design and results of a proof-ofconcept implementation are presented and discussed.
\end{abstract}

\section{INTRODUCTION}

Simple-to-use devices for dielectric permittivity characterization of liquids are highly demanded for chemical, biomedical, and industrial applications. In particular, monitoring the change of a liquid's composition over time in an effort-efficient way is gaining attention. An example is monitoring the healing of wounds after surgery by examining over time the composition of the wound liquid that is drained via a flexible tube. Such change in composition over time is reflected by a change of the dielectric properties (dielectric permittivity and loss tangent) of the liquid-undertest (LUT). Approaches to characterize the dielectric properties of a liquid are already well established, but they are usually off-line and non-continuous, and require expensive measurement equipment, such as a vector network analyzer (VNA). A well-known commercial approach is the coaxial probe, which would be inserted in the LUT, held in a beaker [1]. Such approach is not suitable for practical applications in e.g., hospital settings, as the procedure is time and labor intensive, and requires a highly skilled operator. In this work, we propose an alternative approach based on an oscillator of which its resonator, i.e., a cavity through which the tube passes, is 3D-printed. The latter has the strong benefit that fabrication can be adapted flexibly to local conditions, e.g., tube diameter, and this in an inexpensive way compared to conventional fabrication techniques [2]. The advantage of adopting an oscillator topology is that the dielectric properties of the LUT can be estimated by means of a portable spectrum analyzer. Operating a spectrum analyzer, assuming its power readings have been pre-calibrated, requires less training of the operator, as opposed to the conventional approach consisting of measuring the S-parameters of a coaxial probe or resonator using a VNA. The latter involves the need for frequent VNA recalibrations during usage, involving more training and being more time intensive to execute the measurements. The principle of characterizing the dielectric properties of a LUT by means of an oscillator has been studied already in literature [3]-[5], but these works did not adopt the flexible 3D-printing technique in the resonator fabrication.

This paper is organized as follows. After a summary about the design and the characteristics of the 3D-printed resonator, in Sec. 2, the design and implementation of the oscillator are discussed in Sec. 3. In Sec. 4, experimental results are presented to show that the variation in dielectric properties due to a change in the LUT's composition can be characterized by the 3D-printed resonator based oscillator using a portable spectrum analyzer.

\section{3D-PRINTED RESONATOR}

The cavity resonator with the channel where the LUT flows is designed first. As it can be expected that the quality factor of a 3-D printed resonator is lower than that of a conventional pure metallic cavity resonator, and moreover, 
that it will even be further lowered by the passing of a lossy liquid (i.e., water based) through it, the electrical characteristics of the resonator have to be defined first, before proceeding to the oscillator design.

A thorough review of microwave planar resonator-based sensors was recently presented in [6]. For applications requiring a good compromise between compact size, easy manufacturing and good electrical performance, an interesting example of a 3D-printed cavity resonator with embedded fluidic channel was proposed in [7]: this structure is based on a planar resonant cavity filled with a 3D-printed dielectric material, implemented in substrate integrated waveguide (SIW) technology [8], with a channel for the liquid realized inside the cavity during the printing process. This solution is particularly interesting, as it leads to a compact and planar structure, compatible with integration of other PBC structures, and fabricated by simple printing and metallization processes. However, it presents a low quality factor of the cavity, due to the presence of the dielectric 3D printed material inside the cavity. A mitigation of this issue was proposed in [9], where a partially air-filled SIW cavity [10] was adopted to reduce dielectric losses and increase the quality factor (to the value of 43), while keeping the advantage of the low-profile planar topology. A further increase in the quality factor can be achieved by using fully three-dimensional cavity resonators, where the $3 \mathrm{D}$ printed material is a mere support of the metallization (like the pumpkin-shaped resonator proposed in [11], where the quality factor is increased up to the value of 321), but in this case the compact size and the planar topology of the cavity are lost, making this solution less suitable for the intended applications.

For these reasons, the 3D-printed resonator presented in [9] was selected and adopted in this work, and its structure is briefly summarized here for completeness. The structure consists of a square resonant cavity, with a multi-folded pipe printed inside the cavity, where the liquid is injected (Fig. 1a). Such cavity could be inserted in series of a drainage tube, as used on patients. The design of the internal pipe can be customized to fit the application requirements such as its diameter and folding structure. Even though each change in pipe design implies the need to re-optimize the cavity in order to ensure proper operation of the cavity on the fundamental resonant mode, it does not complicate the corresponding fabrication due to the flexible 3D printing technique. In fact, thanks to manufacturing by stereo-lithography process, the cavity and empty pipe can be realized in one fabrication pass. For the considered 3D-printed resonator, a single material (i.e., photopolymer resin Clear FLGPCL02, with dielectric permittivity $\varepsilon_{\mathrm{r}}=2.9$ and loss tangent $\tan \delta=0.03$ ) was adopted, as this offers full flexibility in the shape and size of both the cavity and the pipe. The adopted stereolithography (SLA) 3D printer guarantees a special resolution of $50 \mu \mathrm{m}$.

The cavity is designed in the form of an SIW cavity, where the side walls are replaced by rows of metal cylinders [8]: in this way, the metallization of the cavity can be realized by top/bottom aluminum foils and screws or rivets for the side walls (Fig. 1b). This metallization procedure appears to be accurate enough for the frequency range considered in this application. The major source of uncertainty in this structure is related to the actual value of the dielectric permittivity of the 3D-printed material: besides the preliminary experimental evaluation of the permittivity, this uncertainty is significantly mitigated by the measurement procedure, which is based on the variation of the resonance frequency and not on the absolute measurement of the resonance frequency. Further details on the fabrication process are documented in [9].

The cavity is designed to operate with the fundamental $\mathrm{TM}_{110}$ mode at the frequency of $3.8 \mathrm{GHz}$ when the pipe is empty. When a liquid is injected in the pipe, the resonance frequency decreases to approximately $3.4 \mathrm{GHz}$ in the case of water. Besides the shift of the resonance frequency of the considered cavity mode, the presence of liquid also modifies the quality factor of the cavity mode, due to the additional losses of the liquid. In [9], the effect of the injected liquid on the mode spectrum of the cavity is discussed, and accurate techniques to determine LUT dielectric parameters from resonant cavity measurements are reported. After the experimental validation of the 3D-printed cavity resonator, reported in [9], the oscillator design can follow.

\section{DESIGN OF LUT-LOADED OSCILLATOR}

The 3D-printed cavity was incorporated as the frequency-selective element in the oscillator design. The oscillator topology is shown in Fig. 2(a). The circuit is based on an Infineon BFP760 BJT transistor in common emitter configuration with emitter degeneration. The 3D-printed resonator is connected through a $50 \mathrm{ohm}$ microstrip line to the base of the BJT, and a positive feedback, formed by a resistor and a capacitor, connects the collector to the base. Finally, the two capacitors $C_{B}$ are used to create DC blocks. 
The oscillator was designed in such a way that its oscillation frequency was strongly dependent on the liquid injected into the cavity. The design was achieved using the commercial software ADS by Keysight. The vendor provided transistor model was implemented and the measured S-parameters of the resonant cavity were imported in the design environment. The S-parameter measurements of other components were imported as well, namely the ones of the right-angle SMA connector, used to connect the 3D-printed cavity to the board, and the board's SMA connectors. The S-parameters of the connectors were obtained by measurements on a separately designed test board and applying the through-line de-embedding algorithm [12]. The circuit was simulated with the harmonic-balance technique. The challenge during the design process was to guarantee the oscillation conditions over the range of expected oscillation frequencies, due to the low quality factor of the LUT-loaded 3D-printed resonator cavity. Therefore, the emitter capacitor $C_{e}$ jointly with the components in the feedback loop had to be properly chosen.

The active circuit was realized in microstrip and its layout is shown in Fig. 2(b). Surface mount device (SMD) components were used for the passive lumped components, and microstrip transmission lines were added to bias the circuit, to create the feedback loop to trigger oscillation operation, and to connect the BJT transistor to the external SMA $50 \mathrm{ohm}$ straight connectors. The lengths of the microstrip lines as well as the values of the SMD components were optimized to obtain oscillations around $4 \mathrm{GHz}$. The values of the SMD components used in the active circuit are listed in the caption of Fig. 2. The design is such that the transistor can be biased in two possible ways: either by connecting both the collector and the base to DC voltage supplies, or just by using a divider to bias the base. The presence of the emitter resistance $R_{e}$ contributes to set the proper collector current. The width of the bias lines was made as narrow as possible, compliant to fabrication limits, as to provide a high characteristic impedance. In addition, quarter wavelength radial stubs on both bias lines have been inserted to block the RF signal at frequencies around $4 \mathrm{GHz}$. The active circuit was realized on a Rogers RO3006 dielectric substrate $\left(\varepsilon_{\mathrm{r}}=6.5\right.$ and thickness 0.64 $\mathrm{mm})$, as shown in Fig. 3(a).

\section{MEASUREMENT RESULTS AND DISCUSSION}

The measurement setup is shown in Fig. 3(b). It consists of a portable N9344C spectrum analyzer (SA) which resolution bandwidth was set to $100 \mathrm{kHz}$. DC bias is applied by two lab sources, which could be replaced by batteries in practical applications. The transistor's DC operation condition was set to a collector current of $30 \mathrm{~mA}$, which corresponded to applying $5 \mathrm{~V}$ and $2.6 \mathrm{~V}$ to the BJT's collector and base, respectively.

As to be able to compare the obtained results with reference measurements, obtained using the commercial coaxial probe technique [1], a set of mixtures was characterized as opposed to studying the time evolution of the LUT passing in the cavity resonator.

The measurements were performed with the same set of mixtures as in [9], namely different concentrations of water and isopropanol (ISP), having dielectric permittivity values spanning from 1 (air) to 76 (water). The highest oscillation frequency $(4.183 \mathrm{GHz})$ is obtained with air, i.e., empty pipe, while the lowest oscillation frequency $(4.103$ $\mathrm{GHz}$ ) is measured when water is injected into the pipe.

To test the performance of the device as a sensor, three materials were selected for calibration, namely air, isopropanol, and water. Their permittivity was determined by standard coaxial probe measurements. The nominal permittivity of these materials versus their measured frequency shift $\Delta f$ (i.e., the difference between the resonance frequency with the empty pipe and the one with the pipe filled with the liquid) is shown by black square markers in Fig. 4(a). For sake of convenience, a fitting function was selected based on the simulation results, with the aim to be simple to use and be accurate enough. The selected formula used to determine the relative dielectric permittivity of the liquid is the following:

$$
\varepsilon_{\mathrm{r}}=a e^{b \Delta f}+(1-a)
$$

where $a$ and $b$ are coefficients to be determined, and $\Delta f$ is expressed in GHz. Based on these three materials, the coefficients of the fitting function were determined, with $a=0.0223$ and $b=100.3$. Subsequently, (1) was adopted to extract the dielectric permittivity of all mixtures, as shown by the small dot markers in Fig. 4(a).

A similar procedure was adopted to extract the loss tangent of the liquids. In Fig. 4(b) the black square markers represent the nominal loss tangent of air, ISP, and water versus the measured output power. Based on these three measurements, a polynomial fitting function was determined: 


$$
\tan \delta=p_{1} \mathrm{P}_{\mathrm{OUT}}^{2}+p_{2} \mathrm{P}_{\mathrm{OUT}}+p_{3}
$$

where $p_{1}=0.4521, p_{2}=-11.05, p_{3}=67.492$, and with $\mathrm{P}_{\text {OUT }}$ expressed in $\mathrm{dBm}$. By applying (2), the loss tangent of the other mixtures was then extracted, as shown by the small dot markers in Fig. 4(b).

The diamond markers in both plots of Fig. 4 represent the dielectric permittivity and loss tangent values determined by standard coaxial probe measurements. These data were used as reference values, with which the permittivity and loss tangent values as obtained by the proposed oscillator are compared.

Table I reports the differences in values of the dielectric permittivity and loss tangent obtained by using the proposed 3D-printed resonator based oscillator with respect to the reference values obtained with the coaxial probe. The stability of the negative sign in the deviations of the permittivity extraction and of the positive sign in the deviations relative to loss tangent extraction is attributed to the ambient temperature difference between coaxial probe and oscillator measurements. The larger disagreements in permittivity of some mixtures (ISP 30\%/water $70 \%$ and ISP $85 \%$ /water 15\%) is probably due to the rapid evaporation of the ISP in some conditions, leading to a different water content in the measurement by coaxial probe and by the proposed system. Unfortunately, due to longterm lab closure imposed by COVID-19 restrictions, these anomalies could not be revisited.

When examining the experimental results, it is noted that the obtained accuracy overall cannot compete with the high accuracy of the coaxial probe method [1], mainly due to the relatively low quality factor of the 3D-printed cavity resonator, and therefore that the proposed design may not be sufficiently suitable for absolute characterizations of a liquid's dielectric properties. Nevertheless, the proposed design is very promising for flexible, in-situ monitoring of a liquid's time-dependent changing composition, as this reverts to examining the relative changes over time.

\section{CONCLUSION}

In this paper, the design of an oscillator based on a 3D-printed cavity resonator is proposed as an operator- and cost-efficient way to monitor in-situ the time evolution of a liquid's dielectric properties. The circuit consists of a 3Dprinted cavity embedding a micropipe, where the presence of a liquid under test modifies the resonance frequency of the cavity and its quality factor. The oscillator, that adopts this cavity as the frequency-selective element, operates at an oscillation frequency that varies with the permittivity of the liquid and with an output power that changes with the loss tangent of the liquid. Its design had to be tailored to meet the oscillation condition over a wide range of liquid compositions, taking into account the 3D-printed cavity resonator's quality factor.

The relationship between the permittivity and the oscillation frequency shift has been determined for this type of oscillator, giving the possibility to extract the LUT's dielectric permittivity. Moreover, the dependence of the oscillator's output power on the loss tangent of the liquid injected in the cavity has been obtained, allowing to extract the LUT's loss tangent.

These results pave the road to other potential applications of liquid characterization at microwave frequencies, that require tailored designs and therefore high manufacturing flexibility, combined with simple characterization operation.

\section{REFERENCES}

[1] D. V. Blackham and R. D. Pollard, "An improved technique for permittivity measurements using a coaxial probe," IEEE Trans. Instrum. Meas., vol. 46, no. 5, pp. 1093-1099, Oct. 1997.

[2] S. A. Nauroze, J. G. Hester, B. K. Tehrani, W. Su, J. Bito, R. Bahr, J. Kimionis, and M. M. Tentzeris, "Additively manufactured RF components and modules: Toward empowering the birth of cost-efficient dense and ubiquitous IoT implementations," Proc. of the IEEE, vol. 105, no. 4, pp. 702-722, Apr. 2017.

[3] O. Elhadidy, M. Elkholy, A. A. Helmy, S. Palermo, and K. Entesari, "A CMOS fractional- $N$ PLL-based microwave chemical sensor with 1.5\% permittivity accuracy," IEEE Trans. Microwave Theory Techn., vol. 61, no. 9, pp. 3402-3416, Sept. 2013.

[4] S. Chen, M. Guo, K. Xu, P. Zhao, Y. Hu, L. Dong, and G. Wang, "A dielectric constant measurement system for liquid based on SIW resonator," IEEE Access, vol. 6, pp. 41163-41172, 2018.

[5] H. E. de Lima Ávila, G. A. de Andrade, F. Rangel de Sousa, and D. J. Pagano, "Modeling and analysis of a PLL-based resonant frequency tracking system using a resonant cavity sensor," IEEE Sensors Journal, vol. 19, no. 17, pp. 7447-7459, Sept. 1, 2019. 
[6] R. A. Alahnomi, Z. Zakaria, Z. M. Yussof, A. A. Althuwayb, A. Alhegazi, H. Alsariera, and N. A. Rahman, "Review of Recent Microwave Planar Resonator-Based Sensors: Techniques of Complex Permittivity Extraction, Applications, Open Challenges and Future Research Directions," Sensors, vol. 21, no. 7, p. 2267, Mar. 2021.

[7] S. Moscato, M. Pasian, M. Bozzi, L. Perregrini, R. Bahr, T. Le, and M. Tentzeris, "Exploiting 3D Printed Substrate for Microfluidic SIW Sensor," 45th European Microwave Conference (EuMC2015), Paris, France, Sept. 7-10, 2015.

[8] M. Bozzi, A. Georgiadis, and K. Wu, "Review of substrate integrated waveguide (SIW) circuits and antennas," IET Microwaves, Antennas and Propagation, vol. 5, no. 8, pp. 909-920, June 2011.

[9] G. M. Rocco, M. Bozzi, D. Schreurs, L. Perregrini, S. Marconi, G. Alaimo, and F. Auricchio, "3D-printed microfluidic sensor in SIW technology for liquids' characterization,” IEEE Trans. Microwave Theory Techn., vol. 68, no. 3, pp. 11751184, Mar. 2020.

[10] F. Parment, A. Ghiotto, T. Vuong, J. Duchamp, and K. Wu, "Air-Filled Substrate Integrated Waveguide for Low-Loss and High Power-Handling Millimeter-Wave Substrate Integrated Circuits," IEEE Trans. Microwave Theory Techn., vol. 63, no. 4, pp. 1228-1238, Apr. 2015.

[11] G. M. Rocco, N. Delmonte, D. Schreurs, S. Marconi, F. Auricchio, and M. Bozzi, "3D-printed pumpkin-shaped cavity resonator to determine the complex permittivity of liquids," Microwave and Optical Technology Letters, 2021 (early view).

[12] K.W. Eccleston, "A new interpretation of through-line de-embedding," IEEE Trans. Microwave Theory Techn., vol. 64, no. 11, pp. 3887-3893, Nov. 2016. 


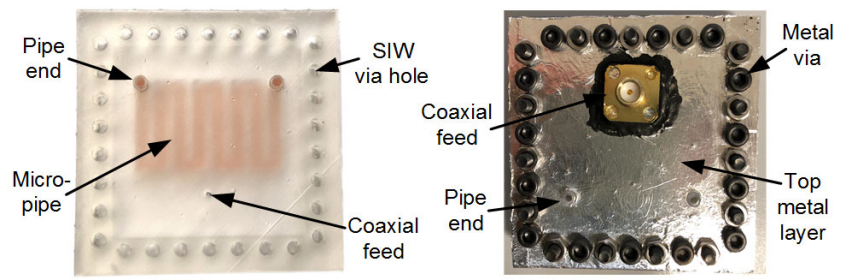

Fig. 1. Photographs of the 3D-printed resonator cavity: (left) Dielectric structure obtained by stereo-lithography process. The pipe is filled by a dark colored liquid for visualization purposes; (right) After metallization. 


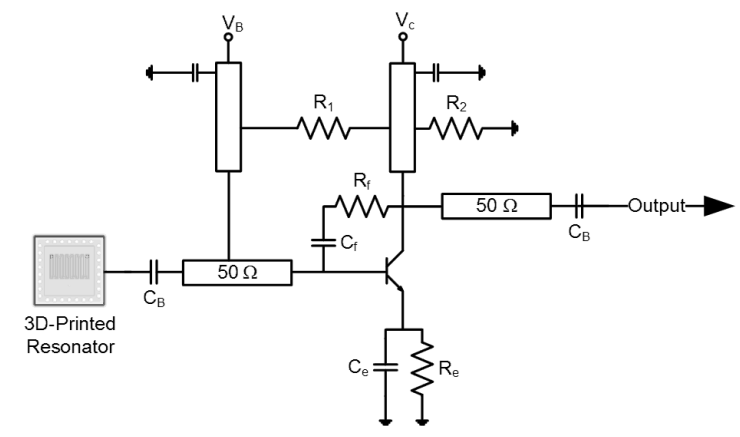

(a)

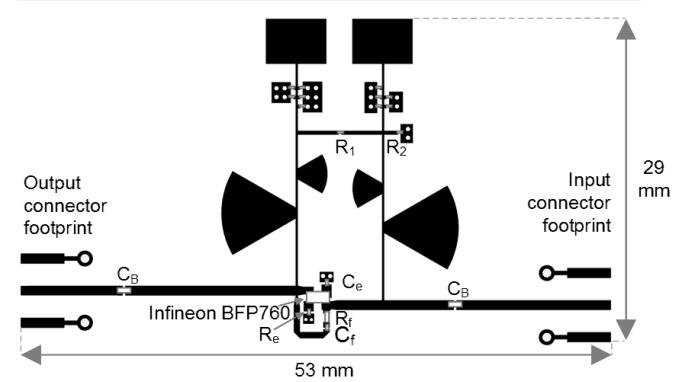

(b)

Fig. 2. Design of the oscillator: (a) Topology; (b) Layout $\left(R_{1}=2.2 \mathrm{k} \Omega, R_{2}=2.7 \mathrm{k} \Omega, R_{e}=62 \Omega, R_{f}\right.$ $\left.=100 \Omega, C_{B}=430 \mathrm{pF}, C_{e}=56 \mathrm{pF}, C_{f}=2 \mathrm{pF}\right)$. 


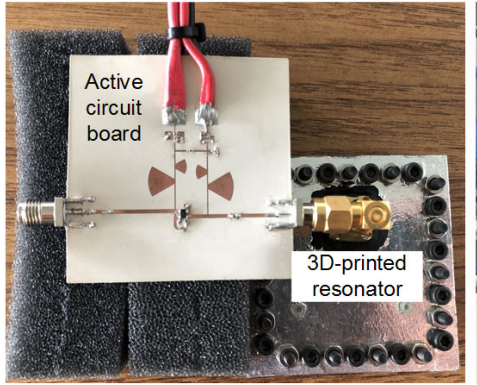

(a)

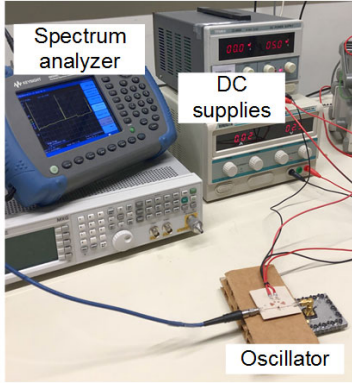

(b)

Fig. 3. Oscillator prototype: (a) Photograph of the circuit connected to the resonator; $(b)$ Photograph of the measurement setup. 


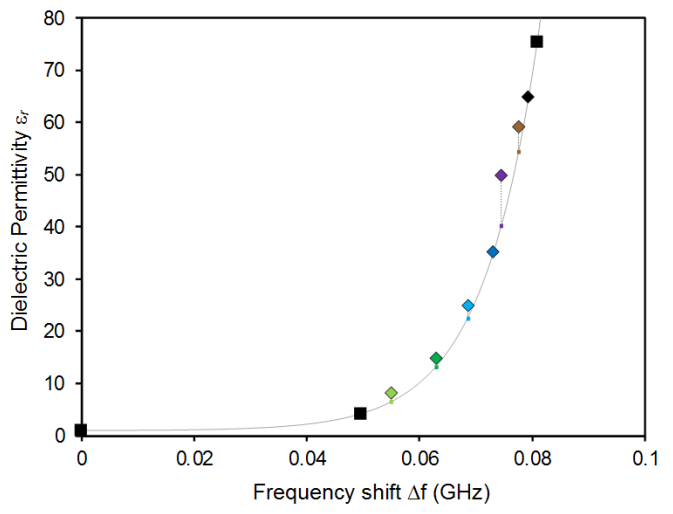

(a)

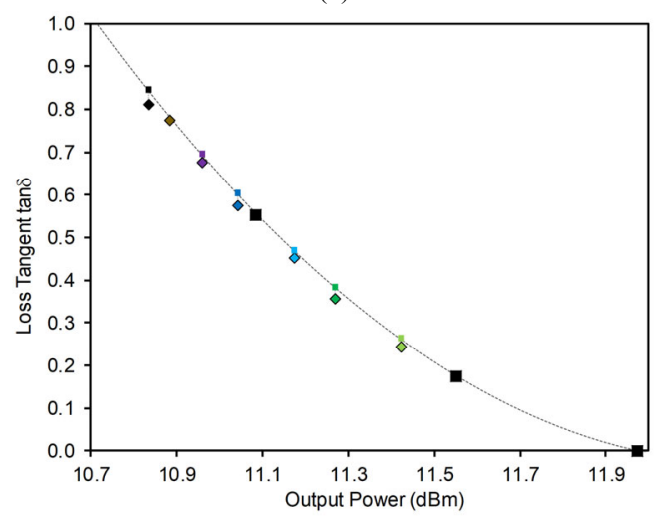

(b)

Fig. 4. Oscillator measurements results used for calibration (black square markers) and extracted values of permittivity/loss tangent (dot markers) compared with the reference values (diamond markers). 


\section{TABLE I}

Dielectric permittivity and loss tangent extracted using (1), (2) and reference values measured with a coaxial probe

\begin{tabular}{|c|c|c|c|c|c|c|c|c|}
\hline \multirow{2}{*}{$\begin{array}{c}\text { LUT } \\
\text { ISP/Water }\end{array}$} & \multirow{2}{*}{$\begin{array}{c}\Delta f \\
(\mathrm{MHz})\end{array}$} & \multirow{2}{*}{$\begin{array}{l}P_{\text {OUT }} \\
(\mathrm{dBm})\end{array}$} & \multicolumn{2}{|c|}{ Oscillator } & \multicolumn{2}{|c|}{ Coax.Probe } & \multicolumn{2}{|c|}{$\begin{array}{l}\text { Error }(\%) \\
\end{array}$} \\
\hline & & & $\varepsilon_{\mathrm{r}}$ & $\tan \delta$ & $\varepsilon_{\mathrm{r}}$ & $\tan \delta$ & $\overline{\varepsilon_{\mathrm{r}}}$ & $\tan \delta$ \\
\hline $10 \% / 90 \%$ & 79.2 & 11.42 & 63.8 & 0.24 & 64.9 & 0.26 & -1.67 & 7.47 \\
\hline $20 \% / 80 \%$ & 77.7 & 11.27 & 55.0 & 0.36 & 59.2 & 0.38 & -7.03 & 7.27 \\
\hline $30 \% / 70 \%$ & 74.5 & 11.18 & 40.2 & 0.45 & 49.9 & 0.47 & -19.44 & 3.50 \\
\hline $45 \% / 55 \%$ & 73.0 & 11.04 & 34.7 & 0.57 & 35.3 & 0.60 & -1.65 & 4.44 \\
\hline $60 \% / 40 \%$ & 68.6 & 10.96 & 22.7 & 0.68 & 24.9 & 0.69 & -8.92 & 2.20 \\
\hline $75 \% / 25 \%$ & 62.9 & 10.88 & 13.2 & 0.78 & 14.8 & 0.78 & -10.61 & 0.83 \\
\hline $85 \% / 15 \%$ & 54.9 & 10.84 & 6.5 & 0.81 & 8.04 & 0.84 & -19.53 & 3.51 \\
\hline
\end{tabular}

